\title{
FINITELY MANY PRIMITIVE POSITIVE CLONES
}

\author{
S. BURRIS AND R. WILLARD
}

(Communicated by Donald Passman)

\begin{abstract}
Given a finite set $A$ there are only finitely many sequences of the form $\left\langle\operatorname{Con}\left(\mathbf{A}^{n}\right)\right\rangle_{n \geq 1}$ or $\left\langle\operatorname{Hom}\left(\mathbf{A}^{n}, \mathbf{A}\right)\right\rangle_{n \geq 1}$, where $\mathbf{A}$ is any algebra on $A$. From this we derive the fact that there are only finitely many primitive positive clones on $A$, which solves a problem posed by A. F. Danil'cenko in the 1970s. Consequently there are only finitely many model companions for universal Horn classes generated by an algebra of a given finite size.
\end{abstract}

This paper is about clones of operations (on a finite set) which are closed under definitions by existentially quantified systems of equations. We call such clones primitive positive clones. ${ }^{1}$

It is reported in [6] that A. V. Kuznecov first defined the notion of a primitive positive clone, and had proved by 1967 that a two-element set has only 25 primitive positive clones. A. F. Danil'cenko [5] subsequently proved that a three-element set has only finitely many primitive positive clones, and she reported [6] in 1979 that the "problem of finiteness" for larger sets was still open.

Our interest in this "problem of finiteness" stems from the study of model companions. In Burris and Werner [4] it is proved that for any finite set $K$ of finite structures the universal Horn class $\operatorname{ISP}(K)$ generated by $K$ has a model companion. Recently $M$. Albert [1] made the fascinating discovery that only finitely many essentially different model companions arise from universal Horn classes generated by a single two-element algebra. This follows from the finiteness result of Kuznecov, plus the following key observation of Albert:

If $\mathbf{A}$ is a finite algebra and $\hat{\mathbf{A}}$ is the expansion of $\mathbf{A}$ to all operations in the primitive positive clone generated by $\mathbf{A}$, then the model companion of $\operatorname{ISP}(\hat{\mathbf{A}})$ is just an extension by definitions (see [9]) of the model companion of $\operatorname{ISP}(\mathbf{A})$.

Indeed the subuniverses of $\mathbf{A}^{I}, I$ arbitrary, which belong to members of the model companion of $\operatorname{ISP}(\mathbf{A})$ are completely determined by the primitive positive clone of A.

In this paper we solve the finiteness problem: namely, we show that for each $k \geq 1$ there are only finitely many primitive positive clones on the set $\{0,1, \ldots, k-$ $1\}$. Consequently, given $k$, there are only finitely many essentially different model

Received by the editors August 15, 1986.

1980 Mathematics Subject Classification (1985 Revision). Primary 08A99; Secondary 03C50.

Key words and phrases. Primitive positive, clone, model companion, universal Horn, congruence.

The first author's research supported by NSERC Grant No. A7256.

The second author's research supported by an Ontario Graduate Scholarship.

${ }^{1}$ Elsewhere they are called parametrically closed classes $[\mathbf{5}, \mathbf{6}]$ or clones acting bicentrally $[\mathbf{1 1}]$. 
companions arising from universal Horn classes generated by a single $k$-element algebra.

1. Preliminaries. Let $A$ be a finite nonempty set. $O_{A}$ denotes the set of all


indexed (rather than nonindexed) algebra on $A$; this is so that we can discuss formulas in the language of $\langle A, \xi\rangle$, and homomorphisms between powers of $\langle A, \mathcal{F}$.

Following A. Robinson, we call a first-order formula of the form $\exists \wedge$ atomic a primitive positive formula. A primitive positive clone on $A$ is a subset $\mathcal{F} \subseteq \bigcirc_{A}$ which contains every operation $f \in O_{A}$ whose graph is definable by a primitive positive formula in the language of $\langle A, \mathcal{F}\rangle$. Clearly every $\mathcal{F} \subseteq O_{A}$ is contained in a smallest primitive positive clone, which we call the primitive positive clone generated by $\mathcal{F}$.

\section{Results.}

THEOREM 1. Let $A$ be a finite set. Then $\left\{\left\langle\operatorname{Con}\left(\mathbf{A}^{n}\right)\right\rangle_{n \geq 1} \mid \mathbf{A}\right.$ is an algebra on $A\}$ is a finite set of sequences.

ProOF. Let $f, g, h, k \in A^{n}$. Then

$$
\langle f, g\rangle \in \Theta_{\mathbf{A}^{n}}(h, k) \quad \text { iff } \quad \mathbf{A}^{n} \models \pi(f, g, h, k)
$$

for some principal congruence formula $\pi(x, y, z, w)$. (Recall [3] that a principal congruence formula is a certain kind of primitive positive formula which witnesses $\mathrm{Mal}^{\prime}$ cev's description of principal congruences.) Note that $\mathbf{A}^{n} \models \pi(f, g, h, k)$ iff

$$
\mathbf{A}=\pi(f(i), g(i), h(i), k(i)) \quad \text { for all } i<n
$$

since $\pi$ is primitive positive. Hence the set $\left\{\pi^{\mathbf{A}} \subseteq A^{4}: \pi\right.$ is a principal congruence formula\} determines the sequence

$$
\left\langle\left\{\Theta_{\mathbf{A}^{n}}(h, k): h, k \in A^{n}\right\}\right\rangle_{n \geq 1}
$$

which in turn determines the sequence $\left\langle\operatorname{Con}\left(\mathbf{A}^{n}\right)\right\rangle_{n>1}$ in the usual way. The theorem now follows from the fact that there are only finitely many sets of 4-ary relations on $A$.

THEOREM 2. Let $A$ be a finite set. Then $\left\{\left\langle\operatorname{Hom}\left(\mathbf{A}^{n}, \mathbf{A}\right)\right\rangle_{n \geq 1} \mid \mathbf{A}\right.$ is an algebra on $A\}$ is a finite set of sequences.

PROOF. Let $\alpha$ be a map from $A^{n}$ to $A$. Then $\alpha$ is a homomorphism from $\mathbf{A}^{n}$ to $\mathbf{A}$ iff

(i) $\operatorname{ker} \alpha \in \operatorname{Con}\left(\mathbf{A}^{n}\right)$, and

(ii) the canonical map $\bar{\alpha}: A^{n} / \operatorname{ker} \alpha \rightarrow A$ defined by $\bar{\alpha}(f / \operatorname{ker} \alpha)=\alpha(f)$ is an embedding of $\mathbf{A}^{n} / \operatorname{ker} \alpha$ into $\mathbf{A}$.

If (i) is true but (ii) is false, there must be a term $t\left(x_{1}, \ldots, x_{m}\right)$ in the language of $\mathbf{A}$, and elements $d_{1}, \ldots, d_{m} \in A^{n} / \operatorname{ker} \alpha$, such that

$$
\bar{\alpha} t^{\mathbf{A}^{n} / \operatorname{ker} \alpha}\left(d_{1}, \ldots, d_{m}\right) \neq t^{\mathbf{A}}\left(\bar{\alpha} d_{1}, \ldots, \bar{\alpha} d_{m}\right) .
$$

Since $\bar{\alpha}$ is injective we can assume (by equating variables) that $m \leq|A|$. Note that $t^{\mathbf{A}^{n} / \operatorname{ker} \boldsymbol{\alpha}}$ is completely determined by $t^{\mathbf{A}}, n$, and $\operatorname{ker} \alpha$. Thus the sequence $\left\langle\operatorname{Con}\left(\mathbf{A}^{n}\right)\right\rangle_{n \geq 1}$ together with the set of at most $|A|$-ary term functions of $\mathbf{A}$ give us 
sufficient information to determine the sequence $\left\langle\operatorname{Hom}\left(\mathbf{A}^{n}, \mathbf{A}\right)\right\rangle_{n \geq 1}$. The theorem now follows from Theorem 1 and the fact that there are only finitely many sets of at most $|A|$-ary operations on $A$.

The preceding theorems have the following sort of consequence. If one is given an algebraic property $P$ of the congruences of an algebra (or of the homomorphisms from one algebra to another) there is an integer-valued function $n_{P}(k)$ such that, for any finite algebra $\mathbf{A}$, we can determine whether all members of the sequence $\left\langle\operatorname{Con}\left(\mathbf{A}^{n}\right)\right\rangle_{n \geq 1}$ (or $\left\langle\operatorname{Hom}\left(\mathbf{A}^{n}, \mathbf{A}\right)\right\rangle_{n \geq 1}$ ) satisfy $\mathcal{P}$ by checking only those for which $n \leq n_{P}(|A|)$. It would be interesting to determine $n_{P}$ for some of the standard properties of congruences like congruence distributive, congruence modular, congruence permutable, skew free, and all congruences are factor congruences.

We now turn to the finiteness problem of primitive positive clones. The following result is credited in the literature $[\mathbf{5}, \mathbf{1 0}]$ to Kuznecov; the first published proof (of an equivalent result) is apparently due to L. Szabó [10]. The result follows directly from the Galois theory for operations and relations on a finite set introduced by Bodnarčuk, Kalužnin, Kotov, and Romov [2] (see also [7]).

Proposition 3. Suppose $\mathcal{F}_{1}, \mathcal{F}_{2}$ are sets of operations on the finite set $A$ and define $\mathbf{A}_{i}$ to be the algebra $\left\langle A, \mathcal{F}_{i}\right\rangle, i=1,2$. Then $\mathcal{F}_{1}$ and $\mathcal{F}_{2}$ generate the same primitive positive clone iff

$$
\left\langle\operatorname{Hom}\left(\mathbf{A}_{1}^{n}, \mathbf{A}_{1}\right)\right\rangle_{n \geq 1}=\left\langle\operatorname{Hom}\left(\mathbf{A}_{2}^{n}, \mathbf{A}_{2}\right)\right\rangle_{n \geq 1} .
$$

COROLlaRY 4. For each finite set $A$, there are only finitely many primitive positive clones on $A$.

PROOF. Combine Theorem 2 and Proposition 3.

A closer look at the proof of Theorem 1 yields the following information. If we write the principal congruence formula $\pi(x, y, z, w)$ as

$$
\exists u_{1} \cdots u_{m} \bigwedge_{i} s_{i}(x, y, z, w, \vec{u})=t_{i}(x, y, z, w, \vec{u})
$$

then we can assume - by equating some of the $u_{i}$ 's without changing $\pi^{\mathbf{A}}$ - that

$$
m \leq|A|^{\left|\boldsymbol{\pi}^{\mathbf{A}}\right|} \leq k^{\left(k^{4}-k^{3}+k^{2}\right)} \quad \text { where } k=|A| .
$$

Hence any primitive positive clone on a $k$-element set is generated by its members of arity at most $k^{\left(k^{4}-k^{3}+k^{2}\right)}+4$. By slightly different methods we can show that any primitive positive clone on a $k$-element set is generated by its members of arity at most $k^{k}$, but this still seems to us to be far from the best possible result (which we conjecture to be $k$ for $k \geq 3$ ).

We remark in closing that our arguments can be easily modified to prove the following. Let $A_{1}, \ldots, A_{r}$ be distinct finite sets, and let $K$ be the set of all finite nonempty products of $\left\{A_{1}, \ldots, A_{r}\right\}$. Then

$$
\left\{\langle\text { Con } \mathbf{B}\rangle_{B \in K} \mid \mathbf{A}_{1}, \ldots, \mathbf{A}_{r} \text { are similar algebras on } A_{1}, \ldots, A_{r}\right\}
$$

and

$\left\{\left\langle\operatorname{Hom}\left(\mathbf{B}, \mathbf{A}_{i}\right)\right\rangle_{B \in K ; 1 \leq i \leq r} \mid \mathbf{A}_{1}, \ldots, \mathbf{A}_{r}\right.$ are similar algebras on $\left.A_{1}, \ldots, A_{r}\right\}$

are finite sets of indexed sets. Hence (see [8]) there are only finitely many sets of $r$-tuples of operations $\left\langle f_{i}: A_{i}^{m} \rightarrow A_{i}\right\rangle_{i=1}^{r}$ which are closed under definitions by 
primitive positive formulas. Consequently there are only finitely many essentially different model companions arising from universal Horn classes generated by $r$ algebras of sizes $\left|A_{1}\right|, \ldots,\left|A_{r}\right|$ respectively.

\section{REFERENCES}

1. M. Albert, A preservation theorem for ec-structures with applications, J. Symbolic Logic (to appear).

2. V. G. Bodnarcuk, L. A. Kalužnin, V. A. Kotov, and V. A. Romov, Galois theory for Post algebras. I, II, Kibernetika (Kiev) 5 (1969), no. 3, 1-10; no. 5, 1-9 (Russian); English transl., Cybernetics 5 (1969), 243-252, 531-539.

3. S. Burris and H. P. Sankappanavar, A course in universal algebra, Graduate Texts in Math., no. 78, Springer-Verlag, New York, 1981.

4. S. Burris and H. Werner, Sheaf constructions and their elementary properties, Trans. Amer. Math. Soc. 248 (1979), 269-309.

5. A. F. Danil'Cenko, Parametric expressibility of functions of three-valued logic, Algebra i Logika 16 (1977), 397-416 (Russian); English transl., Algebra and Logic 16 (1977), 266-280.

6. $\ldots$, On parametrical expressibility of the functions of $k$-valued logic, Finite Algebra and Multiple-Valued Logic (B. Csákány and I. Rosenberg, eds.), Colloq. Math. Soc. János Bolyai, vol. 28, North-Holland, 1981, pp. 147-159.

7. D. Geiger, Closed systems of functions and predicates, Pacific J. Math. 27 (1968), 95-100.

8. R. Pöschel, Die funktionale Vollständigkeit von Funktionenklassen über einer Familie endlicher Mengen, Z. Math. Logik Grundlagen Math. 20 (1974), 537-550.

9. J. Shoenfield, Mathematical logic, Addison-Wesley, Reading, Mass., 1967.

10. L. Szab6, Concrete representation of related structures of universal algebras. I, Acta Sci. Math. (Szeged) 40 (1978), 175-184.

11. _ On the lattice of clones acting bicentrally, Acta Cybernet. 6 (1984), 381-388.

Department of Pure Mathematics, University of Waterloo, Waterloo, OnTARIO, CANADA, N2L 3G1 